\title{
Effects of exercise therapy on postural stability, multifidus thickness, and pain intensity in patients with chronic low-back pain
}

\author{
$\mathrm{R} \mathrm{FINTA}^{1 *}, \mathrm{I}_{\text {POLYÁK }}^{2}, \mathrm{~T}$ BENDER ${ }^{3}$ and E NAGY ${ }^{1}$ \\ ${ }^{1}$ Department of Physiotherapy, Faculty of Health Sciences, University of Szeged, Szeged, Hungary \\ ${ }^{2}$ Affidea Diagnostic Ltd., Szeged, Hungary \\ ${ }^{3}$ Buda Hospital of the Hospitaller Order of Saint John of God, Budapest, Hungary
}

(Received: February 4, 2019; revised manuscript received: May 29, 2019; accepted: June 12, 2019)

\begin{abstract}
Purpose: We examined the effects of exercise therapy on postural stability, multifidus thickness, and pain intensity in patients with low-back pain. Materials and methods: Subjects were divided into a chronic low-back pain (CLBP; $n=10)$ group and a healthy control $(\mathrm{C} ; n=10)$ group. Group CLBP took part in an 8-week training programme, whereas group $\mathrm{C}$ did not. The thickness of the multifidus in both groups was assessed using ultrasonography before and after 8 weeks, in prone and kneeling positions, in relaxed and contracted states. A standing heel-raising test was used to assess postural stability. Results: After the intervention in group CLBP, the thickness of the contracted multifidus increased in the prone position, whereas the thickness of both the contracted and relaxed multifidus decreased in the kneeling position. In group $\mathrm{C}$ after 8 weeks, multifidus thickness decreased in both positions, while both relaxed and contracted. Group C performed the standing heel-raising test significantly better than group CLBP before the 8-week period. After the training, group CLBP improved significantly, but no changes were found in group C. Discussion and conclusions: Changes in thickness of the multifidus correlate with improved postural stability and decreased pain intensity. Decreasing thickness in healthy individuals may be an early sign of developing CLBP.
\end{abstract}

Keywords: postural stability, ultrasonography, low-back pain, atrophy

\section{INTRODUCTION}

Chronic low-back pain (CLBP) affects people in their active ages, and as more and more young people are affected nowadays, it decreases the productivity of working-age generations [1]. CLBP cases can be divided into two groups: those with specific and those with non-specific low-back pain. Specific causes are medical conditions, whereas non-specific CLBP is lumbar pain without a known medical reason or lumbar pain that is not attributed to a specific pathology [2].

\section{Facts about lumbar instability}

Non-specific CLBP may be caused by segmental instability of the lumbar spine [3]. There are many hypotheses, which try to explain what segmental spinal instability is. According to the "neutral zone concept" by Panjabi, a neutral zone and an elastic zone can be differentiated in the total range of movement (ROM). The neutral zone is the initial part of the segmental movement, where internal resistance is minimal, whereas the elastic zone follows after the neutral zone, and the movements occur against internal resistance in the end range of the ROM. When segmental instability emerges, the extent of the neutral zone is increased. Lumbar segmental instability occurs when the capacity of the stabilizer system has decreased, and a normal neutral zone cannot be maintained [4].

\section{Stabilizer subsystems of the lumbar spine}

There are three subsystems responsible for maintaining lumbar stability, namely: the passive, neural, and active subsystems. The passive subsystem is granted, and it is difficult to affect its functioning by non-invasive techniques. Ligaments, tendons, vertebrae, and discs belong to this subsystem. The passive subsystem has the most important role in stabilizing the elastic zone of the ROM. The neural control subsystem has a unique role in the timing of muscle activation corresponding to the given movement [5]. People with low-back pain have reduced postural stability in standing and in sitting positions, and they react worse to disturbing circumstances [6]. Healthy people rely on a multisegmental strategy of postural control to maintain a standing position. They use proprioceptive information from all of their body parts, including the low-back area. When CLBP develops, the neural system reorganizes the sources of the proprioceptive information because of the lack of information from the low-back area. Therefore, people with CLBP mostly use information from the joints of the ankle and apply the ankle strategy more to maintain their postural stability [7]. As a consequence, lumbar pain

\footnotetext{
* Corresponding author: Regina Finta; Department of Physiotherapy, Faculty of Health Sciences, University of Szeged, Temesvári krt. 31, Szeged H-6726, Hungary; E-mail: fintaregina@etszk.u-szeged.hu
}

This is an open-access article distributed under the terms of the Creative Commons Attribution 4.0 International License, which permits unrestricted use, distribution, and reproduction in any medium, provided the original author and source are credited, a link to the CC License is provided, and changes - if any - are indicated. (SID_1) 
influences the body's inclination in the standing position. People with low-back pain lean more forward than the healthy people [7]. Brumagne et al. [8] conducted a study on young people with a short history of low-back pain and compared them to healthy people. They found significant differences between the low-back pain group and the healthy group. When visual input was not available, members of the low-back pain group leaned and positioned the centre of pressure (COP) forward significantly.

The third subsystem is the active subsystem. The active and neural subsystems are primarily responsible for spinal stability in the neutral zone [5]. The muscles' role is prominent in defending the spine from deteriorative impacts. Multisegmental, more superficial muscles are responsible for producing the movements of the trunk. Unisegmental deep muscles function primarily as force transducers, fasten the vertebrae to each other, and provide feedback on spinal position and movements for the neural control subsystem. The lumbar multifidus muscle is one of the most important muscles in segmental control and has a significant role in stabilizing the lumbar spine [5]. Changes of the muscle's morphology, alterations of its function, and the atrophy of this muscle may cause CLBP. The converse can also occur [9].

\section{CLBP and change in muscle function}

Chronic pain causes changes in musculoskeletal functions [10]. Protective adaptation may be the reason for these changes in motor performance, as the pain influences the segmental interneurons. As a result, an increase in muscle tone emerges in people with CLBP. The boost in muscle tone is due to the escalation of stretch reflex activity caused by increased $\gamma$-motoneuron discharge [11]. A pain-spasm-pain model may characterize the neuromuscular adaptation to lumbar pain [10]. The patients' activities are diminished due to pain and stiffness, which result in muscle spasm and strain and eventually this situation aggravates pain in a vicious cycle [12]. Reduced proprioceptive input may cause neuromuscular deficiencies. Such constant malfunctioning of neuromuscular control and flawed regulation of dynamic movements may lead to inappropriate muscular activity (i.e., overutilization or underutilization), which may cause further deteriorations of the muscular system. These symptoms worsen through sensitization of the peripheral and central nervous systems (lowering pain threshold), which promotes dysfunctional movement patterns [12]. Training of the affected muscles can reduce the pain, improve the stabilizer muscle function, and provide normal proprioceptive feedback [13]. CLBP affects muscle function in the lumbar area and causes dysfunction in the lumbar multifidus muscle [14].

The aim of this study was to investigate the effect of an 8-week training programme on the thickness of the lumbar multifidus muscle in patients with CLBP. We monitored the change in thickness in the various stages of pain.

\section{MATERIALS AND METHODS}

\section{Participants}

Young adult participants were recruited from our university, and they were divided into two groups: group $\mathrm{C}$ (control) for asymptomatic individuals and group CLBP for individuals with a history of CLBP (at least 3 months duration of pain [15]). Altogether, 20 subjects were recruited: 10 for group CLBP and 10 for group C. The average age was 20.70 years $(S D=1.49)$ in group CLBP $(n=10)$ and 22.30 years $(S D=1.06)$ in group $\mathrm{C}(n=10)$. The inclusion criteria for group CLBP were CLBP, not undergoing any other treatment during the investigation, and being able to get to the location of the training. The inclusion criterion for group $\mathrm{C}$ was no history of CLBP. Exclusion criteria for both groups were balance problems with a neurological cause, a malignant tumour, a serious organ disease, a previous surgical intervention that affected the trunk, or an inability to cooperate. Because of the tedious measuring process, only physiotherapy students were included in the study, since they have a more developed perception of movement and body awareness based on the characteristics of physiotherapy education. All participants took part voluntarily in the study and gave their written informed consent. The study was in compliance with the principles of the Declaration of Helsinki.

\section{Training}

The members of group CLBP participated in an 8-week complex training programme. There were two training sessions per week (1 hr each). At the beginning of each session, there was a warm-up period and at the end there was a cooldown period, each of $10 \mathrm{~min}$ duration. The main part of the training involved mostly strengthening, stretching, and mobilizing exercises for the muscles of the trunk and the hip. Static and dynamic exercises were performed with the help of tools (e.g., elastic bounds, dumbbells, and heavy balls) and without tools. The programme was completed with balance exercises. Training tools were used to improve members' balance throughout static and dynamic exercises. Three physiotherapists ensured correct implementation of the exercises. Contrary to group CLBP, members of group C did not take part in any training during the 8 -week period.

\section{Measurements}

We measured the thickness of the lumbar multifidus muscle's belly and the intensity of the pain. The pain was assessed in $\mathrm{cm}$, using a Visual Analog Scale [16]. The thickness was measured by B-mode ultrasonography on both sides of the trunk, using a Zonare Z.One Ultrasound System (ZONARE Medical Systems, Mountain View, CA, USA, 2013) in two different positions (prone and kneeling positions) and in two different states (relaxed and contracted states). The thickness was measured by placing the electronic calipers just inside the hyperechoic connective tissue layers.

During measurements in the prone position (lying on the chest with the face down), the curved transducer (with a frequency range between 6 and $2 \mathrm{MHz}$ ) was used longitudinally along the spine, with the midpoint over the L4 spinous process. It was moved laterally and angled slightly medially until the L4/5 zygapophyseal joint could be identified, and the muscle was assessed in a relaxed (calm lying) position and in a contracted state [17]. The students were asked to contract the muscles of the lumbar area without extra movement of the trunk. To reach a more efficient 
muscle contraction, tactile stimulation was implemented above the hypothesized area of the muscle [18].

To examine the postural activity of the muscle, the thickness of its belly was measured in a kneeling position as well. In the kneeling position, the postural function of lumbar multifidus is enhanced due to the vertical position. The students were instructed to keep an erect posture. When they held a quiet kneeling position, it was defined as a relatively relaxed state, and when we asked them for muscle contraction, it was defined as a relatively contracted state. To enhance the contraction caused by the postural activity of the lumbar multifidus muscle, tactile stimulation was applied in the kneeling position as well (Figure 1).

To ensure the same setting for ultrasonography, the skin surface was constantly marked, and the measurement was carried out by the same person with experience in ultrasonography. Test-retest reliability was tested by calculation of intraclass correlation and the reliability coefficient. Both the high interclass correlations (0.991-1.000) and the small repeatability coefficients $(0.008-0.095)$ showed good reliability [19].

For testing the improvement of the lumbar stabilizer system, a modified standing heel-raising test was applied. The test is commonly used to assess the function of plantar flexors, essential muscles for locomotion, and postural tasks [20]. In this study, it was performed on an unstable surface (Dynair) to challenge the postural function of the stabilizer muscles. The participants had to stand in the middle of the disc and raise their heels continuously within $30 \mathrm{~s}$, and the number of the raises was counted. The testing procedure was performed before and after the 8-week period.

\section{Data analysis}

The data analysis and the calculations were executed with Microsoft Office Excel and STATISTICA 13 software (StatSoft, Inc., Tulsa, OK). The Shapiro-Wilk test was used as a normality test. To compare the changes that occurred within one group after 8 weeks, the Wilcoxon Matched Pairs Test was used. To compare the two groups, the Mann-Whitney $U$ test was used. The level of significance was set at $p<.05$.

\section{RESULTS}

Pain

After the training, the severity of the pain decreased from 5.76 to $2.3(\mathrm{~cm})$ in group CLBP $(p=.007)$.

\section{The thickness of lumbar multifidus muscle}

Comparing the before and after data for group CLBP, we found that the thickness of lumbar multifidus muscle increased in the prone position in the contracted state, with a significant difference in the left muscle $(p=.017)$. With group CLBP in the kneeling position, some decrease in the thicknesses was found in every condition, and it was significant in the left muscle in the relatively relaxed state $(p=.009$; Figure 2).

In group $\mathrm{C}$, the thickness of lumbar multifidus muscle decreased in all conditions after 8 weeks. Interestingly, reduction was more marked in the relaxed state than in the contracted state. Significant changes were found in the relatively relaxed states of the muscle in the kneeling position. The extent of the significant decreases in the left muscle $(p=.020)$ and the right $(p=.028)$ were approximately the same (Figure 3).

\section{Modified standing heel-raise test}

Before the 8-week training programme, members of group CLBP performed, on average, $25.80(S D=2.94)$ heel-raises within $30 \mathrm{~s}$, and members of group $\mathrm{C}$ performed 30.70 $(S D=4.32)$. This was a significant difference $(p=.021)$. After 8 weeks, the performance of group CLBP increased significantly $(p=.008)$, as they performed 33.20 $(S D=4.64)$ heel-raises. The members of group $\mathrm{C}$ did not (a)

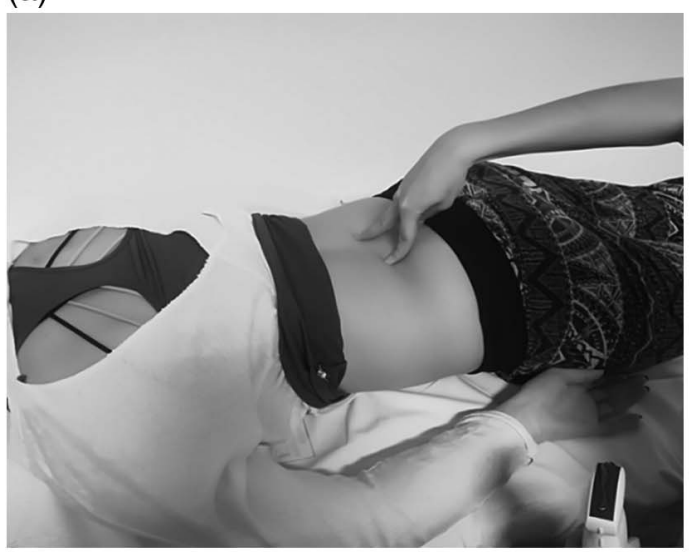

(b)

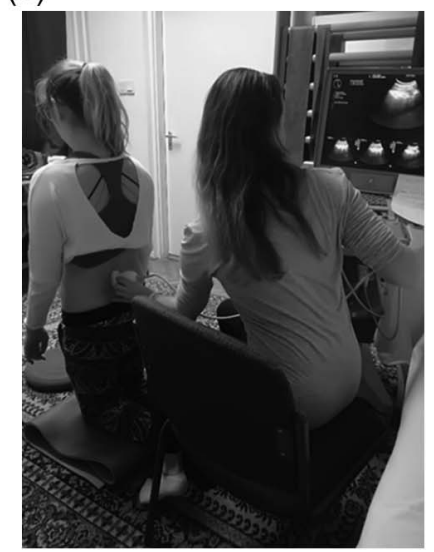

Figure 1. (a) The ultrasonography procedure. The thickness of lumbar multifidus muscle was measured first in a prone position.

The quiet lying was the relaxed state and the contracted state was performed with the help of tactile stimulation on the low-back area. (b) Lumbar multifidus was measured in a kneeling position. During this vertical position, the postural function of the muscle was enhanced in the relatively relaxed state as well. The relatively contracted state of the muscle was performed the same way as in the case of the prone position 


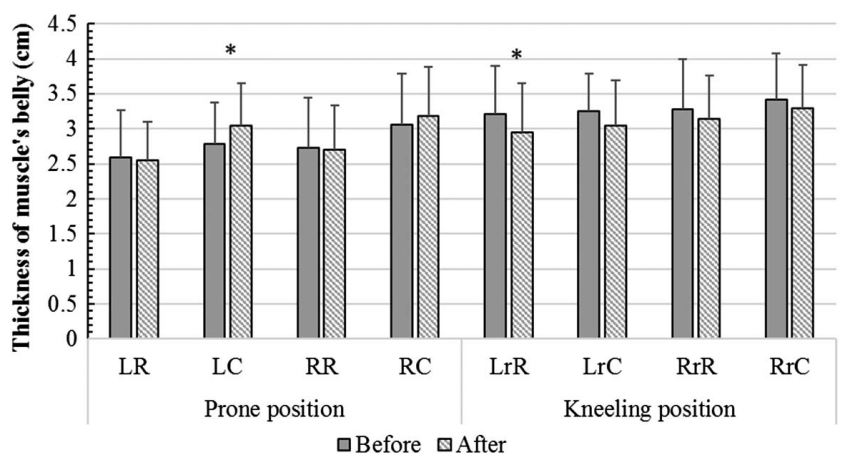

Figure 2. The thickness of lumbar multifidus muscle's belly in case of group CLBP. In the prone position, increased thicknesses of the muscle' belly were found in the contracted states and the thickness of the muscle's belly decreased in every state in the kneeling position. LR: left-sided, relaxed; LC: left-sided, contracted; RR: right-sided, relaxed; RC: right-sided, contracted; r: relatively. $* p<.05$

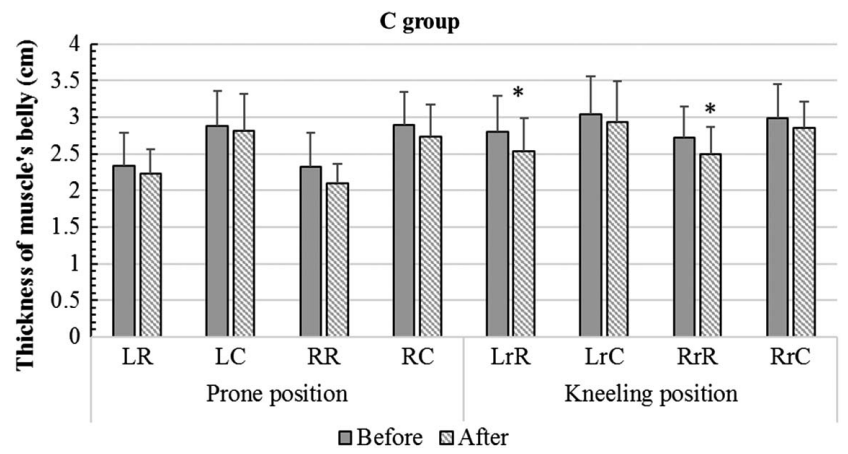

Figure 3. Thickness of lumbar multifidus muscle's belly in group C. Decreases were found in the thickness of the muscle under all conditions. The changes were significant in case of the relatively relaxed states in the kneeling position. LR: left-sided, relaxed; LC: left-sided, contracted; RR: right-sided, relaxed; RC: right-sided, contracted; r: relatively. ${ }^{*} p<.05$

improve their performance significantly $(p=.918)$. The significant difference found between the two groups $(p=.021)$ before 8 weeks vanished by the time of the posttests $(p=.496$; Figure 4$)$.

\section{DISCUSSION}

Our main findings were that during 8 weeks of training for group CLBP, the intensity of pain decreased, postural stability improved, and changes occurred in the thickness of the lumbar multifidus.

\section{Changes in the thickness of lumbar multifidus muscle of group CLBP}

When it was in contraction in the prone position, the thickness of the lumbar multifidus muscle increased after the training. When ultrasonography was performed, both in relaxed and contracted states in the prone position, the muscle's belly was in the neutral state, as in this position, the muscle was not influenced by the enhanced postural

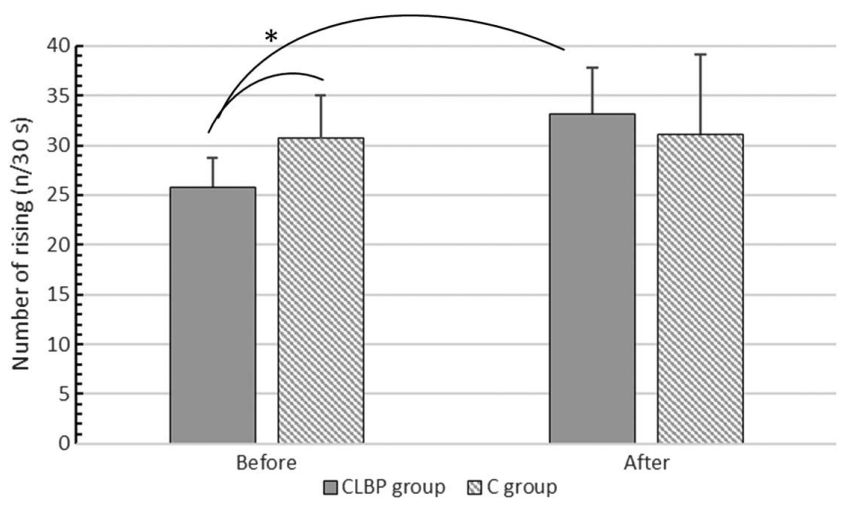

Figure 4. Number of heel-raises before and after 8 weeks. Significant difference was found between the groups during the pretesting procedure. The output of group CLBP improved significantly as a result of the training. There was no change in group $\mathrm{C}$ after 8 weeks. ${ }^{*} p<.05$

function seen in vertical positions [21]. The thicker belly in the contracted state in the prone position indicated the improved contractile ability of the muscle [22]. Considering the facts that the multifidus muscle is smaller in patients with CLBP [23], and that these patients have a reduced ability to voluntarily contract the muscle than healthy people [18], our results indicate that exercise therapy results in a positive change in the condition of the lumbar multifidus muscle in CLBP patients.

Pain can be reinforced by increased co-contraction, which may result in increased stiffness in the lumbar area and altered biomechanical loading [24]. Pain alone can cause an increased muscle thickness [25]. The pain-spasm-pain cycle is a motor control pattern that has the effect of perpetuating the painful disorder, but it also protects the system by maintaining stability on a higher level [26, 27].

Our results showed significant reduction in pain level in group CLBP. For these patients, there was a decreased muscle thickness in the relaxed state in the prone position. However, when voluntary contraction occurred in the prone position, the thickness of the lumbar multifidus muscle belly increased. In the kneeling position, the thickness decreased, even though this posture challenged the multifidus muscle more than the passive prone position [21]. The pain relief may have reduced the strain of the muscle, which resulted in the decrease in its thickness.

We suppose that the changes observed in this study show that the increased co-contraction (in group CLBP) developed by our training programme results in a more normal functioning. With the lumbar pain being relieved by the intervention, the pain-spasm-pain cycle might have been broken [28]. Breaking the pain-spasm-pain cycle may be an explanation for the significant decrease in the thickness of lumbar multifidus muscle in the kneeling position.

To date, conflicting evidence can be found regarding the stabilizer muscles' role and the advantages of strengthening them in CLBP patients, but considering that CLBP is a complex condition [29], it is clear that only one muscle should not be the focus of attention during CLBP management. Therefore, our intervention contains holistic strengthening exercises that activate not only the lumbar multifidus muscle, but also all members of the active stabilizer system 
of the trunk. The members of the stabilizer systems have an influence on each other. Thus, if the function of even one muscle of the active stabilizer system deteriorates, it eventuates an increased demand on the other subsystems to maintain stability [5].

Besides the breaking of the pain-spasm-pain cycle, a further possible explanation for the decrease in the multifidus' thickness in the kneeling position is that in the kneeling position all stabilizer muscles need to be more active to maintain the vertical position of the body than in the passive prone position [21]. Based on this observation, we assume that the activation of the lumbar multifidus was reduced by the neural system as a result of improved function of the whole stabilizer system after the exercise therapy. The increased tension of lumbar multifidus muscle was no longer necessary.

In addition, the observed decrease in the muscle's thickness in the kneeling position in group CLBP can be attributed to a change in posture after our intervention. Former studies proved that individuals with low-back pain prefer the ankle strategy to maintain stability $[12,30]$, and consequently, they lean and position their COP forward [8]. As a result of our intervention, a decreased thickness of the lumbar multifidus muscle's belly was assessed in group CLBP in the vertical, kneeling position. The thicker lumbar multifidus muscle measured before the intervention may be a sign of the forward-leaning position [31] preferred by people with low-back pain [8]. With the reduced pain intensity due to the training programme and the improved stabilizer muscles' function, the postural alignment of the trunk might have changed into a more natural condition [30]. The change of posture from a forward-leaning position to a normal posture influences the functioning of the stabilizer muscles of the trunk [31]. The activity of the muscles in the low-back area is lower in a normal posture than in the forward-leaning position [31] developed by CLBP. After the 8 -week training programme, decreases in the thickness of multifidus were observed by ultrasound examination in group CLBP. Therefore, the observed changes in lumbar multifidus muscle might be an indicator of the recovery of normal posture.

We think that the increase in the thickness of lumbar multifidus muscle during contraction in the prone position and the decrease during relaxation in the prone position, as well as in both contraction states of the vertical, kneeling position were the result of a positive change in group CLBP, which occurred primarily due to the training programme.

\section{Changes in the thickness of the lumbar multifidus muscle in group $C$}

Surprisingly, a decrease occurred in the thickness of lumbar multifidus muscle under all conditions in group C. During the 8 -week period, members of group $\mathrm{C}$ continued their daily routine. Because of the decrease, members of the group were asked if there had been any changes in their daily activities and if they had experienced any pain or stiffness linked to the low-back area during the study. All members of group $\mathrm{C}$ claimed that during the autumn semester, when this study was conducted, they had spent much more time in the sitting position than during the summer vacation before our research began. The students reduced the amount of their physical activity in order to be able to fulfill the requirements of their school. Interestingly, $50 \%$ of group $\mathrm{C}$ developed low-back pain in the last few weeks of the research. Because of the reduced physical activity and the poor posture, generally applied during sitting, atrophy of the lumbar multifidus is presumable. Studies have shown that an altered function and atrophy of the lumbar multifidus muscle can be a cause of CLBP $[5,9]$. Our findings suggest that a decrease in the thickness of the lumbar multifidus muscle may indicate a change in its function. It therefore may be an early sign of developing low-back pain.

\section{Outcomes of the modified heel-raise test}

The results of the modified heel-raise test, which used the unstable Dynair to challenge the postural function of the stabilizer muscles, support our hypothesis that the vicious cycle was broken. People with CLBP have a reduced postural stability, and they react worse to disturbing circumstances [6]. The members of group CLBP showed significant improvement after the intervention, even though they had no chance to practice the test, and there were no exercises for strengthening the triceps surae muscle during the training period. Strengthening of the trunk and hip muscles was emphasized; thus, presumably, the stability of the proximal area (trunk) was improved, thereby facilitating the distal function of heel-raising [32]. Because of the intervention, the severity of pain was reduced, and the stabilizer muscles became stronger, which together resulted in a significant increase in the postural stability of the CLBP patients. In contrast with group CLBP, there was no development in group $\mathrm{C}$.

\section{Limitations}

This study had several limitations. One was the low number of participants, but the recruitment of prospective participants for an extended study is already in progress. Another was that the mechanism of the changes in the thickness of lumbar multifidus muscle due to the training programme was not clarified. The posture and the position of the COP should be measured before and after the intervention, but the applied kneeling position was not suitable for detecting these changes using NeuroCom Basic Balance Master device (Paragon Care Group Pty Ltd., Clayton, Australia). The standing position would be more appropriate to assess the forward-leaning position seen in CLBP patients. It would be beneficial if we could compare the thickness of the lumbar multifidus muscle in CLBP patients to that of the healthy subjects, but the normalizing procedure is not clarified yet [33].

\section{CONCLUSIONS}

The significant results of this study were that, as a result of the applied exercise therapy for group CLBP, changes occurred in the thickness of their lumbar multifidus muscle, their postural stability improved, and their low-back pain 
was relieved. Despite the low sample size, significant changes and clear tendencies were found. However, further research is necessary to investigate the exact reason for these changes. The decreased thickness of multifidus muscle's belly and the simultaneously appearing low-back pain in the healthy individuals draws attention to the importance of lifestyle in the occurrence of low-back pain. Moreover, the decrease in muscle's thickness raises the possibility that this change may be an early sign of developing low-back pain. However, the precise role of the multifidus muscle in CLBP is still unclear [34]. Our investigation mainly focused on lumbar multifidus muscle, but in general CLBP develops as a result of many factors, including social and psychological factors [24]. This study helps to reveal only a few aspects of this complex phenomenon. In conclusion, we can say that the exercise therapy we developed is a viable way to improve muscle function in patients with CLBP. Additionally, the observed changes in muscle function may help in the effort to better understand the altered muscular activation pattern in patients with low-back pain.

Authors' contribution: RF contributed in the conception and design of the study and the acquisition of data. IP designed the ultrasonography. TB and EN drafted and revised the article and share senior authorship.

Ethical approval: The study is in compliance with the principles of the Declaration of Helsinki.

Conflict of Interest/Funding: The authors declare no conflict of interest and no financial support was received for this study.

\section{ABBREVIATIONS}

$\begin{array}{ll}\text { CLBP } & \text { : chronic low-back pain } \\ \text { ROM } & \text { : range of movement } \\ \text { COP } & \text { : centre of pressure } \\ \text { SD } & \text { : standard deviation } \\ \text { C } & \text { : control }\end{array}$

\section{REFERENCES}

1. Allegri M, Montella S, Salici F, Valente A, Marchesini M, Compagnone C, Baciarello M, Manferdini ME, Fanelli G. Mechanisms of low back pain: a guide for diagnosis and therapy. F1000Res. 2016;11(5):1530.

2. Deyo RA, Weinstein JN. Low back pain. N Engl J Med. 2001;344(5):363-70.

3. Panjabi MM. Clinical spinal instability and low back pain. J Electromyogr Kinesiol. 2003;13(4):371-9.

4. Panjabi MM. The stabilizing system of the spine. Part II. Neutral zone and instability hypothesis. J Spinal Disord. 1992;5(4):390-6.

5. Fritz JM, Erhard RE, Hagen BF. Segmental instability of the lumbar spine. Phys Ther. 1998;78(8):889-96.
6. Claeys K, Brumagne S, Dankaerts W, Kiers H, Janssens L. Decreased variability in postural control strategies in young people with non-specific low back pain is associated with altered proprioceptive reweighting. Eur J Appl Physiol. 2011;111(1):115-23.

7. Brumagne S, Janssens L, Knapen S, Claeys K, Suuden-Johanson E. Persons with recurrent low back pain exhibit a rigid postural control strategy. Eur Spine J. 2008;17(9):1177-84.

8. Brumagne S, Janssens L, Janssens E, Goddyn L. Altered postural control in anticipation of postural instability in persons with recurrent low back pain. Gait Posture. 2008;28(4):657-62.

9. Sweeney N, O'sullivan C, Kelly G. Multifidus muscle size and percentage thickness changes among patients with unilateral chronic low back pain (CLBP) and healthy controls in prone and standing. Man Ther. 2014;19(5):433-9.

10. Mense S, Robert DG. Muscle Pain: Understanding the Mechanisms. Berlin, Heidelberg: Springer-Verlag; 2014.

11. Hirayama J, Yamagata M, Ogata S, Shimizu K, Ikeda Y, Takahashi K. Relationship between low-back pain, muscle spasm and pressure pain thresholds in patients with lumbar disc herniation. Eur Spine J. 2006;15(1):41-7.

12. McCaskey MA, Schuster-Amft C, Wirth B, Suica Z, de Bruin ED. Effects of proprioceptive exercises on pain and function in chronic neck- and low back pain rehabilitation: a systematic literature review. BMC Musculoskelet Disord. 2014;15:382.

13. Daman M, Shiravani F, Hemmati L, Taghizadeh S. The effect of combined exercise therapy on knee proprioception, pain intensity and quality of life in patients with hypermobility syndrome: a randomized clinical trial. J Bodyw Mov Ther. 2019;23(1):202-5.

14. Freeman MD, Woodham MA, Woodham AW. The role of the lumbar multifidus in chronic low back pain: a review. PM\&R. 2010;2(2):142-6.

15. Duthey B. Low back pain. In: Priority Medicines for Europe and the World "A Public Health Approach to Innovation". Update on 2004 Background Paper; 2013. p. 4-23.

16. Huskisson E. Measurement of pain. Lancet. 1974;304(7889): $1127-31$.

17. Kiesel KB, Uhl TL, Underwood FB, Rodd DW, Nitz AJ. Measurement of lumbar multifidus muscle contraction with rehabilitative ultrasound imaging. Man Ther. 2007;12(2):161-6.

18. Wallwork TL, Stanton WR, Freke M, Hides JA. The effect of chronic low back pain on size and contraction of the lumbar multifidus muscle. Man Ther. 2009;14(5):496-500.

19. Finta R, Nagy E, Bender T. The effect of diaphragm training on lumbar stabilizer muscles: a new concept for improving segmental stability in the case of low back pain. J Pain Res. 2018;11:3031-45.

20. Svantesson U, Herbert-Losier K, Wessman C, Alricsson M. The standing heel-rise test: reference values and test-retest reliability for healthy individuals from 20 to 81 years of age. Physiotherapy. 2015;101(1):1458.

21. O'sullivan PB, Grahamslaw KM, Kendell M, Lapenskie SC, Möller NE, Richards KV. The effect of different standing and sitting postures on trunk muscle activity in a pain-free population. Spine (Phila Pa 1976). 2002;27(11):1238-44.

22. Zhang S, Xu Y, Han X, Wu W, Tang Y, Wang C. Functional and morphological changes in the deep lumbar multifidus using electromyography and ultrasound. Sci Rep. 2018;8(1):6539. 
23. Fortin M, Macedo LG. Multifidus and paraspinal muscle group cross-sectional areas of patients with low back pain and control patients: a systematic review with a focus on blinding. Phys Ther. 2013;93(7):873-88.

24. O'sullivan P. Common misconceptions about back pain in sport: Tiger Woods' case brings five fundamental questions into sharp focus. Br J Sports Med. 2015;49(14):905-7.

25. Koppenhaver S, Harris D, Harris A, O'Connor E, Dummar M, Croy T, Walker M, Flynn T. The reliability of rehabilitative ultrasound imaging in the measurement of infraspinatus muscle function in the symptomatic and asymptomatic shoulders of patients with unilateral shoulder impingement syndrome. Int $J$ Sports Phys Ther. 2015;10(2):128-35.

26. Hodges PW, Cholewicki J, Van Dieën JH. Spinal control: the rehabilitation of back pain: state of the art and science. Physiother Can. 2015;67(4):394.

27. Lee HJ, Lim WH, Park J-W, Kwon BS, Ryu KH, Lee JH, Park YG. The relationship between cross sectional area and strength of back muscles in patients with chronic low back pain. Ann Rehabil Med. 2012;36(2):173-81.

28. Langevin HM, Sherman KJ. Pathophysiological model for chronic low back pain integrating connective tissue and nervous system mechanisms. Med Hypotheses. 2007;68(1): 74-80.

29. Lederman E. The myth of core stability. J Bodyw Mov Ther. 2010;14(1):84-98.

30. Janssens L, McConnell AK, Pijnenburg M, Claeys K, Goossens N, Lysens R, Troosters T, Brumagne S. Inspiratory muscle training affects proprioceptive use and low back pain. Med Sci Sports Exerc. 2015;47(1):12-9.

31. Floyd WF, Silver PHS. The function of the erectores spinae muscles in certain movements and postures in man. J Physiol. 1955;129(1):184-203.

32. Willson JD, Dougherty CP, Ireland ML, Davis IM. Core stability and its relationship to lower extremity function and injury. J Am Acad Orthop Surg. 2005;13(5):316-25.

33. Linek P, Saulicz E, Wolny T, Myśliwiec A. Body mass normalization for ultrasound measurements of adolescent lateral abdominal muscle thickness. J Ultrasound Med. 2017;36(4):775-82.

34. Wong AYL, Parent EC, Funabashi M, Stanton TR, Kawchuk GN. Do various baseline characteristics of transversus abdominis and lumbar multifidus predict clinical outcomes in nonspecific low back pain? A systematic review. PAIN ${ }^{\circledR}$. 2013;154(12):2589-602. 